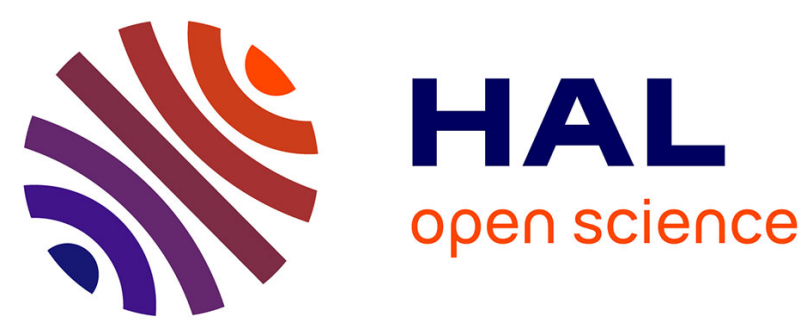

\title{
OPTICAL THEORY-BASED SIMULATION OF ATTENUATED TOTAL REFLECTION INFRARED SPECTRA OF MONTMORILLONITE FILMS
}

Brian Gregoire, Baptiste Dazas, Maud Leloup, Fabien Hubert, Emmanuel Tertre, Eric Ferrage, Sabine Petit

\section{To cite this version:}

Brian Gregoire, Baptiste Dazas, Maud Leloup, Fabien Hubert, Emmanuel Tertre, et al.. OPTICAL THEORY-BASED SIMULATION OF ATTENUATED TOTAL REFLECTION INFRARED SPECTRA OF MONTMORILLONITE FILMS. Clays and Clay Minerals, 2020, 68 (2), pp.175-187. 10.1007/s42860-020-00073-x . hal-03000917

\section{HAL Id: hal-03000917 https://hal.science/hal-03000917}

Submitted on 24 Nov 2020

HAL is a multi-disciplinary open access archive for the deposit and dissemination of scientific research documents, whether they are published or not. The documents may come from teaching and research institutions in France or abroad, or from public or private research centers.
L'archive ouverte pluridisciplinaire HAL, est destinée au dépôt et à la diffusion de documents scientifiques de niveau recherche, publiés ou non, émanant des établissements d'enseignement et de recherche français ou étrangers, des laboratoires publics ou privés. 
OPTICAL THEORY BASED SIMULATION OF ATTENUATED TOTAL REFLECTION INFRARED SPECTRA OF MONTMORILLONITE FILM

Brian Grégoire*, Baptiste Dazas, Maud Leloup, Fabien Hubert, Emmanuel Tertre, Eric Ferrage, Sabine Petit.

Institut de Chimie des Milieux et Matériaux de Poitiers (IC2MP), Université de Poitiers, CNRS, F-86073 Poitiers, France. 
Infrared analyses of clay minerals samples are usually performed by transmission techniques. While transmission measurements are easy and inexpensive, the sample preparation plays a primordial role in the quality of the data. Alternatively, attenuated total reflection (ATR) provides a powerful and often simpler analysis method. Attenuated Total Reflectance, Clay minerals, numerical simulation, infrared optical constants. confusion in the interpretations. Indeed, optical effects play a prominent role in the ATR spectral profile and their identification is mandatory for obtaining quantitative information regarding molecular/particle orientation or film thickness. Here, exact spectral simulations of montmorillonite films are performed by making use of the optical theory, following the determination of the anisotropic optical constants from the experimental reflectance spectra by Kramers-Kronig transformation. This methodology can advantageously be used (i) to choose the appropriate optical conditions for advanced and reliable characterization of clay minerals, (ii) to extract quantitative information such as the estimation of the film thickness, and (iii) to discriminate optical phenomena (optical interferences) from chemical/structural features of the sample.

\section{KEYWORDS}

4 
The chemistry of mineral surfaces and the processes taking place thereon in aqueous environment play a pivotal role in a wide spectrum of chemical, physical and geological processes (Stumm 1997; Lambert 2008; Polubesova and Chefetz 2014). The large surface area in combination with the high retention capability give to clay minerals important adsorption performances for organics and metal ions (Brigatti et al. 2013). To date, probing interfacial processes remains a challenging task for experimentalists because of the complexity of the system. Surface analytical techniques usually require separating the solid from the aqueous solution prior to analysis with the undesirable consequence of completely changing the surface characteristics of the solid or the adsorbed species. Though such methods may be helpful in providing some insights into the nature of the interface under investigation, comparison with the batch experiments may be ambiguous. With the constant effort given by experimentalists to integrate molecular data into macroscopic adsorption models extracted from isotherm measurements, it is of prime importance to probe the interface in-situ for capturing an accurate picture of the interactions and surface modifications occurring in real systems.

In this context, attenuated total reflectance Fourier transform infrared spectroscopy (ATR-FTIR) is particularly valuable as a molecular spectroscopic technique sensitive to both the inorganic and organic parts, and with the possibility of adapting diffusion cells on the top of the internal reflection element (IRE) for probing in-situ dynamical processes (Hind et al. 2001; Lefèvre et al. 2012). Although the transmission method is usually preferred for characterizing materials using the Beer Law, the dispersion of the refractive index and the interference phenomena are commonly ignored. In addition, the delicate task of preparing pellets of samples homogeneously diluted in a transparent matrix suitable for transmission analysis contributes to the frequency variations commonly observed in literature for similar systems (Iglesias et al. 1990). Unlike transmission, ATR gives a quasi-absorption spectrum which is similar but not identical to transmission (Yamamoto and Ishida 1994). This technique greatly facilitates sample preparation and the recorded signal is usually enhanced with respect to transmission enabling the measurements of single inorganic or organic layers. However, interpretation of ATR spectra is more difficult because of the prominence of optical effects (Hansen 1965, 1968). A direct consequence of these effects is the apparent shift of the vibrational bands and intensity variations when compared to transmission spectra making the assignment of vibrational bands more challenging.

Given this strong connection between band profiles and instrumental setup used for analyses, infrared spectra should be regarded as a sample "fingerprint" only for a given condition. The most relevant information that can be obtained from IR measurement is the optical constants of the sample which are independent of the measurement 
methods and allow cross-referencing with results obtained under different experimental conditions (Tolstoy et al. 2003; Hasegawa 2017). Optical constants describe phenomenologically the interaction of the radiation with the matter, providing insight into the molecular structure of a material. Extracting the optical constants from the spectroscopic observables is not straightforward. Two different approaches have been adopted for extracting the optical constants from reflectance spectra of clay minerals. The first one is based on Kramers-Kronig transform to analyze normal or quasi-normal reflectance data. For example, Karakassides et al. performed Kramers-Kronig analysis on reflectance spectra at $10^{\circ}$ off-normal incidence to compute the optical and dielectric properties of montmorillonite clay minerals in pellet form (Karakassides et al. 1997). These analyses enable to evidence the migration of $\mathrm{Li}^{+}$cations into the layer structure upon thermal treatment. Alternatively, some authors model the optical constants of phyllosilicate minerals by making use of dispersion theory using non-linear least square optimization routines and a best-fit approach (Glotch and Rossman 2009; Roush et al. 1991; Esposito et al. 2000). This approach consists in defining the vibration in a crystal lattice as a sum of the vibrations of numerous oscillators leading to dispersion equations associating the complex dielectric function as a function of the frequency. The optical constants, $\mathrm{n}(v)$ and $\mathrm{k}(v)$, are then directly determined from the dielectric function. The slight discrepancy usually observed in the determined optical constants by these two approaches likely originates from the sample preparation and the calculation methods. Optical constants are therefore macroscopic parameters, and can be hardly transferable if acquisition or sampling conditions are not identical.

The determination of optical parameters is a prerequisite for advanced interpretation of structural features based on optical spectroscopy. As an example, Ras and coworkers carried out numerous studies to determine the structure and orientation of hybrid organo-clay preparations by various infrared spectroscopic methods (Ras et al. 2003, 2004b, 2004c, 2007b). These authors successfully prepared highly oriented monolayer films of clay minerals by the Blodgett-Langmuir method, enabling them to investigate the orientation and conformation of organic molecules adsorbed onto the clay minerals by attenuated total reflectance spectroscopy. Orientational determination consists in relating changes in intensity of a vibrational band obtained with the electric field oriented parallel or perpendicular to the plane of incidence to the average angle between the transition moment at the origin of this vibrational band and the electric field of the infrared radiation. These calculations require the determination of the mean squared electric field amplitudes which need the sample optical constants as input parameters. For the reasons explained above, the correctness of the orientational order determination relies strongly on the sample optical constants. 
These examples illustrate that macroscopic properties such as the orientational order of a given sample are transferable only if all the input parameters enabling the determination of that property are determined on the same preparation. Motivated by this statement, this work aims at providing ATR users with the necessary equations for computing the optical constants from polarized ATR measurement that can be further used for the quantitative interpretation of infrared spectra. The determination of the anisotropic optical constants is based on a KramersKronig transform analysis applied to polarized ATR spectra of thick Na - SWy-3 montmorillonite film (Bardwell and Dignam 1985; Dignam and Mamiche-Afara 1988; Bertie and Lan 1996). These optical constants are then used to simulate spectra under a variety of conditions. Because the orientational order has already been treated in the literature (Grégoire et al. 2020), this manuscript specifically focuses at the determination of film thickness and its influence on the measured spectra, the absorption coefficient and the qualitative influence of the presence of water.

The benefit of this methodology is two-fold. First, and for a sample with given optical constants, the simulated spectra can reveal the effects of interferences due to e.g. thickness, orientation, etc. First, the calculations being based on optical theory, the simulated ATR spectra only evaluate optical interferences because the sample property are described by their experimental optical constants. Therefore, deviations between the experimental and calculated spectra may originate from changes in the sample property (such as porosity or orientation) giving evidence for specific interactions or changes in sample chemistry which affect optical properties. Second, it can help at choosing the most relevant experimental conditions for the acquisition of high-quality spectra such as the appropriate IRE element, angle of incidence, polarization angle, film thickness, optical configurations (e.g. internal or external reflectance, normal or off-normal transmission, etc.).

\section{THEORETICAL BACKGROUND}

Spectral simulation

The development of the equations for extracting the optical constants treat the system as a series of stratified parallel optical boundaries as depicted in Figure 1. The propagating radiation can pass through $\mathrm{n}$ phases, where phase $\mathrm{j}$ is described by the complex refractive index $\widehat{n}_{J}(v)$ given by :

$$
\widehat{n}_{j}(v)=\sqrt{\widehat{\epsilon}_{j}}(v)=n_{j}(v)+i k_{j}(v)
$$

Where $n_{j}(v)$ is the real refractive index of the medium in phase $\mathrm{j}, k_{j}(v) k \kappa$ is the absorption index and $\hat{\epsilon}_{j}(v)$ is the related complex dielectric function. To simplify the notation, the frequency dependence of the optical constants will be deliberately omitted. In an ATR experiment as depicted in Figure 1, phase 1 corresponds to the ATR IRE which is optically described by a constant real refractive index assuming no absorption of the incident 
electromagnetic radiation $\left(n_{1}=4\right.$ for Germanium IRE and $n_{1}=2.4$ for Diamond and ZnSe IRE). Phase 2 corresponds to the sample film, optically defined by a complex optical constant. In the case where the sample thickness is below the penetration depth of the evanescent wave, the optical constants of phase 3 needs to be explicitly described i.e. assuming air $\left(n_{3}=1\right)$ or water (defined with complex $\left.\widehat{n_{3}}\right)$ for example. The extent to which radiation is reflected or transmitted at an interface depends on the light polarization, the angle of incidence of the incoming radiation and the optical constants of the phases at the interface. The observable in an ATR experiment is the reflectance spectrum, which is determined from the Fresnel amplitude coefficient, $\hat{r}_{j k}^{i}$ :

$$
R_{i}=\left|\hat{r}_{j k}^{i}\right|^{2}=\frac{E_{r}^{2}}{E_{\text {inc }}^{2}} \text { with } i=s, p
$$

The script $i$ refers to the polarization direction which is either $s$ when the electric field vector is perpendicular to the incident plane or $p$ for a parallel electric field vector. The Fresnel coefficients are defined as the ratio of the complex amplitude of the electric field vectors of the reflected waves, $E_{r}$, to the one of the incident waves, $E_{\text {inc. }}$. They can be calculated at the interface between phases $\mathrm{j}$ and $\mathrm{k}$, if the incident angle to this interface, $\alpha_{\mathrm{j}}$, and the optical constants of phase $\mathrm{j}$ and $\mathrm{k}$ are known according to :

$$
\hat{r}_{j k}^{S}=\frac{\xi_{j}-\xi_{k}}{\xi_{j}+\xi_{k}} \text { and } \hat{r}_{j k}^{p}=\frac{\hat{n}_{k}^{2} \xi_{j}-\hat{n}_{j}^{2} \xi_{k}}{\hat{n}_{k}^{2} \xi_{j}+\hat{n}_{j}^{2} \xi_{k}}
$$

Where $\xi_{j, k}^{i}=\hat{n}_{j, k} \cos \alpha_{j, k}=\sqrt{\left(\hat{n}_{j, k}^{2}-n_{1}^{2} \sin ^{2} \alpha_{1}\right)}$ can be regarded as the refractive coefficient for phase $\mathrm{j}$ or $\mathrm{k}$. Here, $\alpha_{1}$, corresponds to the angle of incidence which is set at $45^{\circ}$ in our study. Equations 2 and 3 allow to compute the reflectance spectra of a two-phase system, i.e. when the thickness of the sample filmed on the ATR IRE is higher than the evanescent wave penetration depth (few microns). In the case where a thin film is deposited on the ATR IRE, more than one interfaces have to be described and the incident radiation undergoes multiple reflections at each interface. Therefore, the amplitude of the reflected radiation, $\widehat{r_{123}, p}$, is calculated by the summation of the reflected rays of the multiple reflection at each interface.

$$
\widehat{r_{123}^{s, p}}=\frac{\widehat{r_{12}^{S, p}}+r_{23}^{\widehat{S, p}} \exp \left(2 i \delta_{s, p}\right)}{1+r_{12}^{\widehat{S, p}} \widehat{r_{23}^{S, p}} \exp \left(2 i \delta_{s, p}\right)}
$$

Where $\delta_{i}=\frac{2 \pi * d * \xi_{2}^{i}}{\lambda}$ indicates the change of the phase of the wave between phase 2 and 3 which depends on the film thickness, $d$. Therefore, the reflectance spectra of a three phase system, $R=\left|\frac{\widehat{r_{123}^{S, p}}}{r_{13}^{S, p}}\right|^{2}$ can be computed for various thicknesses, $\mathrm{d}$, if $\widehat{n_{j}}$ of the three phases are known. For total reflectance, $\widehat{r_{13}, p}=1$, the reflectance spectra 
161 for the s-polarisation : $I_{S}=-2 \arctan \frac{\sqrt{n_{1}^{2} \sin ^{2} \alpha_{1}-n_{\infty}}}{n_{1} \cos \alpha_{1}}$

Determination of the optical constants of uniaxial thick film. number that can be written with polar coordinate as : Mamiche-Afara 1988) :

$$
\text { for the p-polarisation : } I_{p}=-2 \arctan \frac{n_{1} \sqrt{n_{1}^{2} \sin ^{2} \alpha_{1}-n_{\infty}}}{n_{x, y} n_{z} \cos \alpha_{1}}
$$

for ATR geometry is therefore easily obtained from equation 4. It is noteworthy that this method can be generalized to anisotropic layers. In this case, $\hat{n}_{j}$ must be replaced by $\hat{n}_{y}$ and $\hat{n}_{x}$ for $\mathrm{s}$ and p polarization, respectively. The refractive index for the p polarization become, $\xi_{j}^{p}=\sqrt{\frac{\left(\hat{n}_{x}^{2}-\hat{n}_{x}^{2} n_{1}^{2} \sin ^{2} \alpha_{1}\right)}{\hat{n}_{z}^{2}}}$. Therefore, spectral simulations can also be carried out for uniaxial $\left(\hat{n}_{x}=\hat{n}_{y} \neq \hat{n}_{z}\right)$ and biaxial symmetry $\left(\hat{n}_{x} \neq \hat{n}_{y} \neq \hat{n}_{z}\right)$.

The following method is applicable only if the stratified model is composed of a single interface, and therefore, the thickness of the sample is larger than the penetration depth (few microns thick). In the condition of total reflection when the angle of incidence is greater than the critical angle, $\left(\xi_{j}^{i}\right)^{2}=\left(\hat{n}_{j}^{2}-n_{1}^{2} \sin ^{2} \alpha_{1}\right)$ become negative even for non-absorbing sample $\left(k_{j} \rightarrow 0\right)$ and therefore the Fresnel equation (Equation 3) becomes a complex

$$
\hat{r}_{j k}^{i}=\left|\hat{r}_{j k}^{i}\right| \exp \left(i \delta_{i}\right)=R_{i}^{1 / 2} \exp \left(i \delta_{i}\right)
$$

Where $\delta_{\mathrm{i}}=\arg \left(\hat{r}_{j k}^{i}\right)$ correspond to the phase shift. Therefore, in the ATR mode, the incident electric fields undergo phase shifts upon reflection even for non-absorbing sample, in contrast to external reflection at normal incidence. Given that $\hat{r}_{j k}^{i}$ is function of the optical constants $n_{j}$ and $k_{j}, \ln \left(\mathrm{R}^{1 / 2}\right)$ and $\delta_{\mathrm{i}}$ must satisfy the Kramers-Kronig relations. This method was successfully applied by Karakassides et al. for recovering the optical constants from the external reflectance spectra obtained at quasi-normal incidence (Karakassides et al. 1997). Unlike normal reflectance experiments, in the case of ATR and because of the phase shift induced by reflections under total reflection conditions, the $\delta_{\mathrm{i}}$ determined from $\ln \left(\mathrm{R}_{\mathrm{i}}{ }^{1 / 2}\right)$ has to be corrected (Plaskett and Schatz 1963; Bardwell and Dignam 1985; Dignam 1988; Dignam and Mamiche-Afara 1988; Ohta and Ishida 1988; Buffeteau et al. 1999; Boulet-Audet et al. 2010). Dignam et al showed that this correction factor, $I_{\mathrm{s}, \mathrm{p}}$, takes a particularly simple form that can be readily estimated. The phase shift for the ATR geometry is therefore expressed as (Dignam and

$$
\delta_{S}\left(v_{a}\right)=\mathrm{I}_{S}-\frac{2}{\pi} \mathrm{P} \int_{0}^{\infty} \frac{v \cdot \ln \left(\mathrm{R}_{\mathrm{s}}\right)^{1 / 2}}{v-v_{\mathrm{a}}} \mathrm{d} v \text { and } \delta_{p}\left(v_{a}\right)=\mathrm{I}_{p}-\frac{2}{\pi} \mathrm{P} \int_{0}^{\infty} \frac{v \cdot \ln \left(\mathrm{R}_{\mathrm{p}}\right)^{1 / 2}}{v-v_{\mathrm{a}}} \mathrm{d} v
$$


where $n_{\infty}$ represents the refractive index of the sample at infinite wavenumber in a region of zero absorbance. This value was estimated based on a best fit approach between the simulated and experimental ATR spectra for various film thicknesses. The best agreement was obtained by setting $n_{\infty}=1.4$. The calculation of the phase shift according to Equation 6 requires reflectivity data over the complete frequency range, zero to infinity. In practice, the spectra are obtained on a limited frequency range. Therefore, the truncation at high and low wavenumber imposes a limitation on the accuracy achievable using the KK transformation but should be relatively small because the experimental values of $\ln \left(\mathrm{R}^{1 / 2}\right)$ at the cut-off wavenumbers are very small and constant. The KK transformation was numerically implemented by the Maclaurin formula providing a better accuracy than the double Fourier transform (Ohta and Ishida 1988; Huang and Urban 1992). The Fresnel reflection coefficient was then calculated from Equation 5 and the in-plane, $\widehat{\varepsilon_{x y}}$ and out-of-plane, $\widehat{\varepsilon_{z}}$ complex dielectric function was obtained through the following relations:

$$
\begin{gathered}
\widehat{\varepsilon_{x y}}(v)=n_{1}^{2}\left[\sin ^{2} \alpha_{1}+\left(\frac{1-\widehat{r_{s}(v)}}{1+\widehat{r_{s}(v)}}\right)^{2} \cos ^{2} \alpha_{1}\right] \\
\widehat{\varepsilon_{z}}(v)=\frac{n_{1}^{2} \sin ^{2} \alpha_{1}}{\left[1+\left(\frac{\overline{\varepsilon_{x y}}}{n_{1}^{2}}\right)\left(\frac{1-r \overline{p(v)}}{1+r \overline{p(v)}}\right)^{2} \cos ^{2} \alpha_{1}\right]}
\end{gathered}
$$

Then, $\hat{n}_{x}, \hat{n}_{y}$ and $\hat{n}_{z}$ are directly determined using Equation 1. It is noteworthy that using solely the s-polarized spectra for the KK transform according to the corresponding equations allows the determination of the isotropic optical constants.

\section{MATERIALS AND METHODS}

\section{Clay minerals}

The SWy-3 montmorillonite was obtained from the Source Clays Repository of the Clay Minerals Society. The $<1 \mu \mathrm{m}$ size fraction was collected by centrifugation and Na-saturated using three saturation cycles in a $1 \mathrm{M} \mathrm{NaCl}$ solution. The excess of salt was removed by dialysis until $\mathrm{Cl}$ - anion was no more detected by $\mathrm{AgNO}_{3}$ test. $\mathrm{The}$ concentration of the resulting suspensions of Na-SWy-3 montmorillonite is $24.7 \mathrm{~g} / \mathrm{L}$.

\section{Spectral acquisition}

All spectra were recorded with a Nicolet IS50 Fourier transform infrared spectrometer (ThermoScientific) equipped with a DLaTGS detector, and a KBr beamsplitter. ATR spectra were acquired with the VeeMaxIII accessory (Pike Technologies) fitted with a $45^{\circ}$ angle germanium IRE having a crystal diameter of $20 \mathrm{~mm}$ and 
giving a penetration depth of $650 \mathrm{~nm}$ at $1000 \mathrm{~cm}^{-1}$ (assuming $n_{2}=1.4$ ). The germanium IRE was thermoregulated at $25^{\circ} \mathrm{C}$ for all experiments. The angle of incidence, $\alpha_{1}$, was set to $45^{\circ}$. The electric field of the incident infrared beam was polarized either perpendicular (s) or parallel (p) to the plane of incidence using a $\mathrm{ZnSe}$ wire grid polarizer placed in the optical beam path before the sample compartment. Given that for a $45^{\circ}$ incidence angle and isotropic samples, the intensity ratio $\mathrm{Ap} / \mathrm{As}=2$, the efficiency of the polarization was checked on a methanol solution deposited on the ATR IRE and tightly covered to prevent evaporation. In our experimental setup, the Ap/As ratio was measured to be 1.97 suggesting an efficient polarization of the electric field. To compensate for this deviation, a factor of 1.016 was applied to all p-polarized spectra.

Spectra were acquired with a $4 \mathrm{~cm}^{-1}$ resolution and 100 scans were co-added in the range $4500-400 \mathrm{~cm}^{-1}$ using a Happ-Genzel apodization. The spectra were never smoothed or deconvolved. The only correction was a baseline adjustment in some cases. Background spectra were acquired in the same condition as for sample using $\mathrm{s}$ and $\mathrm{p}$ polarized radiation.

Spectral simulation

The computed program for the calculation of ATR spectra of anisotropic layers was realized using MatLab@ (Software version R2017b). Some input parameters had to be introduced such as the incidence angle set at $45^{\circ}$, the electric field polarisation (i.e. s or p), the number of layers (i.e. 2 or 3), and the IRE refractive index (2.4 and 4 for diamond and germanium IRE, respectively).

\section{Film preparation}

The simulation of ATR spectra under various experimental conditions required the prior determination of the optical constants of the SWy -3 Montmorillonite film. A volume of $500 \mu \mathrm{L}$ of the stock dispersion $(24,7 \mathrm{~g} / \mathrm{L})$ was firstly deposited on the Ge IRE and allowed to dry at $25^{\circ} \mathrm{C}$. Then, s and p polarized spectra were acquired. This methodology ensures that the thickness of the sample film is much larger than the penetration depth (few microns thick). The as-obtained s and p-polarized spectra are then used for the extraction of the anisotropic optical constants $\left(\hat{n}_{x}=\hat{n}_{y} \neq \hat{n}_{z}\right)$ using Equations 5,6 and 7.

The as-determined optical constants are further used for the simulation of ATR spectra on films of various thicknesses which are compared to experimental ones. The thickness of the experimental film, d, was estimated based on the Na-SWy-3 Montmorillonite formula, assuming one layer of water as expected at room temperature and ambient humidity, corresponding to an estimated density of $2.3 \mathrm{~g} / \mathrm{cm}^{3}$. The germanium IRE having a surface 
area of $314 \mathrm{~mm}^{2}$, a volume of $100 \mu \mathrm{L}$ was pipetted from diluted suspensions prepared from the $\mathrm{SWy}-3$ stock dispersion and was dropped on the IRE surface for targeting film thicknesses ranging from about 7 to $230 \mathrm{~nm}$. Because the germanium surface is hydrophobic, the original aqueous suspension was diluted by methanol resulting in better wettability and ensuring homogeneous film thickness over the IRE surface. When the methanol was fully evaporated and the sample was equilibrated to ambient humidity, $s$ and $p$ polarized spectra were acquired. The estimated thickness was input in the model for the simulation of the ATR spectra. Based on a best fit approach, a variation of $10 \%$ of the targeted d value was allowed accounting for changes in surface rugosity and porosity and to compensate for measurement precision and accuracy during suspension preparation.

For one experiment, a film with an estimated thickness of $14 \mathrm{~nm}$ was firstly deposited on the Ge IRE, and washed with deionized water several times until no more changes in the vibrational intensity of the SWy-3 film were observed. ATR spectral simulation was performed by adjusting the thickness of the sample in the simulation until qualitative good match is obtained between the experimental and calculated intensity of the clay mineral film. The best fit was obtained with an input thickness of $7 \mathrm{~nm}$. In order to evaluate the influence of water on the top of clay film, a drop of deionized water was added and polarized spectra were acquired where the corresponding polarized background spectra was only bulk water.

\section{RESULTS AND DISCUSSION}

In order to confirm the optical anisotropy of clay platelet, FTIR transmission measurement was firstly performed. The absorption intensity of an infrared radiation relies on a scalar product of the vibrational transition moment amplitude and the amplitude of the electric field of the radiation. Accordingly, absorption bands are observable only if the angle between the electric vector and the transition moment is different from $90^{\circ}$. In the case of oriented samples measured through transmission at normal incidence with s- and p-polarized radiation, only transition moments within the plane of the absorbate result in absorption (Margulies et al. 1988). The transmission spectra of Na-SWy-3 sample oriented by drop-casting on a ZnSe substrate and acquired at normal incidence using polarized radiation in the spectral range $700-1400 \mathrm{~cm}^{-1}$ (Fig. 2a), show the vibrational signatures of the clay layers. Roughly, the region between 700-950 corresponds to hydroxyl bending vibrations from the octahedral sheet, whereas the region $1000-1400 \mathrm{~cm}^{-1}$ is usually assigned to Si-O stretching vibration of the tetrahedral sheet (Farmer and Russell 1964). For both s- and p-polarization, the absorbance spectra are similar, suggesting that the in-plane transition moments are isotropically distributed within the plane of the sample. Taking advantage of the Berreman effect, tilting the sample with respect to the radiation at an angle of $40^{\circ}$ enables the out-of-plane transition moment to be excited (Berreman 1963). The absorbance spectra (Fig. 2b) display noticeable 
differences around $1085 \mathrm{~cm}^{-1}$ that correspond to a transition moment almost perpendicular to the film plane. Samples with transition moments that are isotropically distributed within the sample plane and anisotropically distributed in the perpendicular direction are called transverse isotropic systems. As a consequence, a proper description of the optical properties of clay minerals requires the determination of the optical constants in an uniaxial symmetry $\left(\widehat{n_{x y}} \neq \widehat{n_{z}}\right)$.

\section{Determination of anisotropic optical constants from polarized ATR measurements}

The advantages of ATR geometry enable the computation of both the in-plane $\widehat{n_{x y}}$ and out-of-plane optical constants $\widehat{n_{z}}$ on a thick film of Na-SWy-3 montmorillonite sample with a single preparation. As stated in the introduction, the Fresnel reflection coefficients were determined from the experimental ATR reflectance spectra (Fig. 3a) by taking advantage of the Kramers-Kronig relationship (Equation 5,6). As a result, no fitting procedure is required because all calculations are based on the recorded reflectance spectra and the ATR experimental setup. While the dielectric function described the response of the materials to an electromagnetic radiation, the exact resonance frequency of vibrational modes cannot be accessed experimentally. Indeed, even if the position of reflectance bands is close to the wavelength of absorption, a shift in position is expected to occur accounted for porosity, particle shape and size that affect the dielectric function. The dielectric function determined from the Fresnel coefficients using Equations 7 has to be considered as a macroscopic function that may be different from the microscopic dielectric function which depends only on the resonant vibrational frequency of an IR-active mode. Both macroscopic and microscopic dielectric properties can be connected using an effective medium approach although retrieving the intrinsic dielectric functions from the measured ATR spectra and the Fresnel reflection coefficient is a challenging task. Alternatively, a modelling strategy has been developed by Balan et al for calculating the ATR spectra of powder samples by ATR spectroscopy (Balan et al. 2002, 2011; Aufort et al. 2016). It consists in calculating the ATR spectra from an oscillator model using vibrational mode frequencies determined by first principle quantum mechanics to derive the microscopic dielectric functions. An approximate macroscopic dielectric function is then calculated by considering that the substance of interest is embedded in a matrix which is described by its own dielectric property. Given that the determined macroscopic dielectric properties is a "fingerprint" of the sample being analyzed, the reported optical constants in this study are solely those of the film which is likely different from the mineral itself. The in-plane $\widehat{n_{x y}}$ and out-of-plane optical constants $\widehat{n_{z}}$ (Fig. 3b,c) was determined from the anisotropic dielectric function using Equation 1. The optical 
derivative shape at the maximum intensity of the $k$ spectrum. Given that the determination of $\widehat{n_{x y}}$ and $\widehat{n_{z}}$ has never been performed on clay minerals in the infrared region, comparison with already published optical constants can only be achieved by averaging the anisotropic optical constants to obtain the isotropic optical constants $\widehat{n_{2}}$. The $\widehat{n_{2}}$ value determined in this work are slightly different from those already published based on the DrudeLorentz dispersion theory (Roush et al. 1991; Esposito et al. 2000; Glotch and Rossman 2009). Given that the optical constants are always determined from the recorded reflectance spectra, it is very likely that the discrepancies originate from the sample preparation and the calculation methods.

Strong dispersion of $n_{x y}$ associated with intense absorption of $k_{x y}$ is observed in the Si-O stretching region at $1039 \mathrm{~cm}^{-1}$. the anomalous dispersion of the $n_{x y}$ curve reaches a maximum value of 2.73 at $1004 \mathrm{~cm}^{-1}$ (Fig. $3 \mathbf{b}$ ). Recalling that total reflection occurs only when the incident angle is greater than the critical angle, one may calculate the maximum value that $n_{2}$ can reach to satisfy this condition. For Ge IRE $\left(\mathrm{n}_{1}=4\right)$ and assuming an incident angle of $45^{\circ}$, the maximum $n_{2}$ value is 2.83 while for diamond IRE $\left(\mathrm{n}_{1}=2.4\right)$ this value was found to be 1.70. Because the experimental value of 2.73 is below the maximum value for Ge IRE, the condition of total reflection is respected for both polarizations and within the whole wavelength range investigated. Using diamond or ZnSe IRE is expected to be more problematic for reliable ATR measurements because the condition of total reflection is no more fulfilled and the critical angle will be higher than the incident angle in the wavenumber range $850-1030 \mathrm{~cm}^{-1}$. It is therefore predictable that frequency shift, or distorted band would appear on the ATR spectra when using a low refractive index crystal like diamond or $\mathrm{ZnSe}$. The easiest and more convenient way to reduce these effects is to select high refractive index crystal, such as Ge IRE, to ensure that the angle of incidence will be well above the critical angle (Boulet-Audet et al. 2010). It is noteworthy that verifying the optical conditions for ATR measurement is a prerequisite for quantitative analysis of ATR spectra. Edgar 1980) and to its direction (Fraser and MacRae 1973). Therefore, all the information about particle orientation is inherently included in the in-plane, $k_{x y}$ and out-of-plane, $k_{z}$ extinction coefficients. Examining Fig. 3c, vibrations in the range $1000-1200 \mathrm{~cm}^{-1}$ assigned to tetrahedral sheet transition moments do not present clear orientation because each vibrational mode includes in-plane and out-of-plane components. The bending of the hydroxyl groups in the octahedral sheet decomposes in four different vibrations usually assigned to $\mathrm{AlAlOH}$, FeAlOH, MgAlOH and $\mathrm{MgFeOH}$ located at 919, 883, 842 and $800 \mathrm{~cm}^{-1}$ respectively. These bands are highly responsive to polarization with some having mainly in-plane $(\mathrm{MgAlOH}$ and $\mathrm{AlAlOH})$ or out-of-plane $(\mathrm{Fe} \mathrm{AlOH}$ 
and $\mathrm{MgFeOH}$ ) transition moments in good agreement with the results from Ras et al (Ras et al. 2003). These authors rationalized their results by suggesting that the orientation of the hydroxyl groups depend on the nature of the nearest cations in the octahedral sheets. Although the nature of the octahedral cations may substantially influence hydroxyls orientations, these modes are likely coupled with other vibrations in the tetrahedral units that may alter their responses toward polarization (Larentzos et al. 2007).

\section{Spectral simulations from the determined optical constants}

Now that phase 2 in Fig. 1 is optically defined by the determined $\widehat{n_{x y}}$ and $\widehat{n_{z}}$ values on Na-SWy-3 film, exact spectral simulations can be performed to examine the spectral profiles under various conditions. Given that ultra-thin film in combination to surface-sensitive techniques can provide valuable information about interface phenomena, emphasis is given to the influence and benefits of different optical configurations (e.g. transmission, internal or external reflections, etc.) that could give complementary information or improved sensitivity when used together. Focusing more specifically to ATR methods, the spectral simulation allows to estimate the sample thickness and to highlight changes on the spectral profile as due to the variation of the sample thickness. On a final example, the influence of water or air as the top layer on the ATR spectra will be quantitatively investigated.

Choosing the appropriate optical configuration for Na-SWy-3 thin film analysis: Because the wavelength of infrared radiation $(2-20 \mu \mathrm{m})$ is much greater than the dimensions of the crystal unit cell, only long-wavelength lattice modes interact with the incident light. These optical modes are described with respect to the phonon propagation direction: the transverse optical modes, $\mathrm{TO}$, in which the atomic displacements are perpendicular to the direction of the periodicity of the wave-vector and the longitudinal vibration (LO) where the displacements are parallel to the wave vector. The frequencies of the LO and TO phonon modes are defined with respect to the intrinsic dielectric function. Because of the macroscopic nature of the dielectric function obtained through the KK transform, only the $\mathrm{TO}_{\mathrm{i}}$ and $\mathrm{LO}_{\mathrm{i}}$ energy loss functions, defined as imag $\left(\widehat{\varepsilon_{l}(v)}\right)$ and $\mathrm{imag}\left(\frac{-1}{\overline{\varepsilon_{l(v)}}}\right)$, respectively are accessible, and described the TO and LO surface modes of a thin crystallite layer (Fig. 4a,b). They are conveniently used for describing the spectral shape depending on the optical configuration regardless of whether the TO and LO phonon modes are known. Therefore, the determination of the TO and LO energy loss functions could be a precious help for adequately choosing the appropriate optical configuration thanks to the various surface selection rules observed on thin film. For example, simulated spectra under various optical configurations assuming a film thickness, d, of $20 \mathrm{~nm}$ was calculated with Equation 2-4 (Fig. 4c-g). Because of the transverse nature of electromagnetic radiation, infrared optical measurement of uniaxial oriented thin film using either s-and 
p-polarized light only activates in-plane $\mathrm{TO}_{\mathrm{xy}}$ and $\mathrm{LO}_{z}$ surface modes from surface-parallel or surfaceperpendicular transition moments whichever the experimental setup used. Therefore, the $\mathrm{TO}_{\mathrm{xy}}$ and $\mathrm{LO}_{z}$ energy loss function are plotted along the calculated spectra to better identify the nature of the transition moment involved as a function of the optical configuration. As far as normal transmission is concerned, the simulated spectrum profile is close to the $\mathrm{TO}_{\mathrm{xy}}$ energy loss function and therefore, this optical method activate only surface-parallel transition moments, independently of the polarization (Fig. 4c). In a very similar way, the same information can be obtained from external reflectance measurement at normal (or near-normal) incidence (Fig. 4d). These two optical methods are sometimes used for calculating the in-plane optical constants $\hat{n}_{x y}$. (Berreman 1963) demonstrated that acquisition of IR transmittance spectra at off normal incidence allowed the activation of LO surface modes using p-polarized radiation (Fig. 4e). Indeed, at oblique incidence, the p-polarized radiation gives rise to two components that are parallel and perpendicular to the film surface and hence, excites both $\mathrm{TO}_{\mathrm{xy}}$ and $\mathrm{LO}_{\mathrm{z}}$ surface modes respectively. Both surface-parallel and surface-perpendicular components of the transition moment are then activated and the spectral profile is therefore a combination of the $\mathrm{TO}_{\mathrm{xy}}$ and $\mathrm{LO}_{z}$ energy loss functions. Using spolarized radiation enables to record only surface-parallel component of a transition moment very similarly to what is obtained using classical transmission techniques. This is very similar to ATR spectroscopy, and further demonstrates the benefit of this technique for extracting anisotropic optical constant or for carrying out orientational measurements (Fig. 4e,f). Reflection-absorption at grazing angle on thin film deposited on metal substrate may be of interest because in this condition the p-polarized radiation couple with the surfaceperpendicular component of the transition moment (Ras et al. 2004a). The spectra therefore is essentially driven by the $\mathrm{LO}_{z}$ energy loss function (Fig. $\mathbf{4 g}$ ). Only the most classical optical configurations are illustrated here. However, the methodology based on the calculations of the Fresnel coefficient enable to determine the best experimental conditions simply by changing some variables like film thickness, incidence angle, etc.

Estimation of sample thickness and its influence on the ATR spectra: From the determination of the in-plane and out-of-plane optical constants of the studied montmorillonite, infrared spectra of multi-layer systems can be computed for a variety of incidence angle, polarization, layer thickness conditions using the formalism detailed in the theory section. The benefit of such simulations lies in a better understanding of the origin of the experimental spectra profile, and allows to evaluate whether the observed spectral changes originate from optical effects or chemical effects resulting from specific interaction or ordering. To illustrate this point, clay films of different thicknesses were deposited on the IRE surface and the polarized spectra were acquired and compared to the calculated ones where the estimated thickness was input in the model (Fig. 5). To account for potential changes in 
particle orientation as a function of the film thickness, the s- and p-polarised ATR spectra, $A_{s}$ and $A_{p}$, were converted to isotropic spectra, $\mathrm{A}_{0}$, according to the relation:

$$
A_{0}=\frac{A_{p}+2 A_{s}}{3}
$$

The calculated spectra and the experimental ones are in a fairly good agreement suggesting that the calculation method of $\hat{n}_{x y}$ and $\hat{n}_{z}$ is accurate (Fig. 5). Thus, the variation in position of the vibrational bands account mainly for optical intereferences within the film thickness. Deviations between the calculated and experimental spectra may arise from the surface rugosity and probably from the variation of film porosity for different thicknesses which are not explicitly taken into account in the simulations.

Using the $n_{2}$ and $k_{2}$ optical constants, s- and p- polarised ATR spectra were simulated for various film thicknesses (Fig. 6). In this condition, the orientation of the clay particles is reflected in the determined anisotropic optical constants and implicitly taken into account. The spectral profiles for s- and p- polarization display appreciable variations as the thickness of the clay film increases. The origin of these changes is only attributed to optical effects which depends on the surface to volume ratio of the film as well as the electric field amplitude along the film thickness (Harrick 1965). Interestingly, a mode appears for thin film at $1085 \mathrm{~cm}^{-1}$ which shifts to lower wavenumber when the thickness increases. The frequency of this mode is correlated to the $\mathrm{LO}_{\mathrm{z}}$ surface modes of Si-O describing the vibration of the silicon with apical oxygen (Fig. $4 \mathbf{b})$. This mode being strongly z-oriented has been used to determine the orientation of particle with respect to the laboratory frame (Johnston and Premachandra 2001; Ras et al. 2003, 2007a; Amarasinghe et al. 2008).

This mode shifts linearly to lower wavenumber when the film thickness increases in the range 1-150 nm (Fig. 7a). If an electromagnetic radiation propagates along an oriented film (and the electric field is perpendicular to the film surface), positive ions and negative ions will be displaced to one and the other extremity of the film respectively. This separation of charges creates an additional electric field perpendicular to the film plane whose frequency varies with the incident wave frequency. This charge displacements contribute to an additional restoring force with respect to their equilibrium position. This force can be assimilated to the one perpendicular to the front of a longitudinal wave. Therefore, the frequency of this transverse wave will be close to the LO surface modes. When a thick film is deposited on an IRE, the frequency of LO surface modes decreases and shifts toward the TO surface modes because the contribution of surfaces charges to the created internal field decreases. When the electric field is parallel to the film plane (the wave propagates in the normal direction to the film surface), no additional field 
arises because the crystal is considered as infinite plane, so the frequency corresponds to that of the transverse vibration in the infinite crystal.

Note that the intensity of this LO surface mode also varies with the film thickness modifying the dichroic ratio (As/Ap) although the orientation of the particle is unchanged. As a consequence, the sample film thickness needs to be carefully controlled if the determination of particle orientation in thin clay film is targeted. In order to experimentally determine the film thickness, the most intense $\mathrm{Si}-\mathrm{O}$ stretching mode at $1039 \mathrm{~cm}^{-1}$ can be used. The absorbance is linearly correlated to the film thickness in a rather narrow range from 1 to $100 \mathrm{~nm}$ (Fig. 7b). This contrasts with transmission measurements where the absorbance is proportional to the concentration of particles. The methodology presented here based on the prior determination of $\widehat{n}_{x y}$ and $\widehat{n}_{z}$ thus represents an efficient way to estimate the film thickness (Fig. 5). Alternatively, a modified form of Beer-Lambert law accounting for reflectivity is often used in literature.

$$
-\log R=\alpha d_{e}
$$

The application of this equation requires the determination of the Naperian absorption coefficient, $\alpha$, and the effective thickness, $d_{e}$. The effective thickness introduced by Harrick is defined as the hypothetical thickness that would result in the same absorbance value in a transmission measurement as obtained from ATR experiment (Harrick and Beckmann 1974). The effective thickness is actually much greater or much less than the actual film thickness because it only represents an equivalent pathlength in a transmission spectrum. Owing to the exponential decay of the electric field along the film thickness, the determination of the electric field amplitude need to be estimated (Harrick 1965). The approximate equations proposed by Harrick for the determination of the electric field amplitude are only applicable for very weak absorbers ( $k$ tends to zero) which is not the case of the Si-O stretching vibrations (Fig. 3c).

The Napierian absorption coefficient, $\alpha$, is a more reliable parameter that can be directly compared to transmission measurements. It is defined by :

$$
\alpha_{S i-O}^{S}=4 \pi k_{y} v
$$

In order to enable comparison with transmission measurement, and recalling that only in-plane vibrational modes are accessible at normal incidence transmission, the extinction coefficient, $k_{y}$, has to be selected. From Equation 29, the experimentally determined absorption coefficient for the Si-O band is $2.94 \times 10^{4} \mathrm{~cm}^{-1}$. For comparison, a value of $3.60 \times 10^{4} \mathrm{~cm}^{-1}$ were reported by Johnston (Johnston and Premachandra 2001). These 
authors determined the absorption coefficient by transmission method where the Na-SWy-3 montmorillonite film thickness was evaluated by weighting the dried residue on an IR transparent substrate of known dimensions. The slight difference in the determination of the absorption coefficient with these two methods is likely due to particle orientations. Note that the methodology presented here requires the preparation of a single sample, whose thickness is controlled by the limited penetration depth of the evanescent wave. Therefore, the sample thickness can be assumed as homogeneous through the sample, well above the penetration depth for the entire wavelength range. For a $1 \mathrm{~nm}$ thick film, corresponding to the thickness of a unique smectite layer, intensity of the $\mathrm{Si}-\mathrm{O}$ vibration is calculated to be $1.6 \times 10^{-3}$ which confirms the value of $1.5 \times 10^{-3}$ determined by Johnston et al. and Ras et al. by transmission measurements (Johnston and Premachandra 2001; Ras et al. 2003). As suggested by these authors, single layer of smectite is measurable through modern FTIR spectrometer, where the sensitivity is in the range 1 $\mathrm{x} 10^{-5}$. The very similar results obtained by ATR and transmission methods clearly demonstrates that ATR spectroscopy is a versatile method enabling quantitative measurements.

Contribution of surrounding medium to ATR spectra: air and water: The benefit of ATR spectroscopy lies in the possibility of in-situ analysis of dynamical processes. Typically, a liquid cell is mounted on the ATR IRE where a thin film of a material was previously deposited on. Solutions of interest are then injected through the liquid cell and spectra are recorded to monitor the interactions between the film and the solute (Schampera and Dultz 2009; Lefèvre et al. 2012; Schampera et al. 2016). For quantitative analysis of these interactions, the effect of the solution on top of the sample on the ATR spectra of materials has to be determined. Intuitively, the presence of water instead of air on top of the deposited film at the IRE surface contributes to the variation of the Fresnel reflection coefficient which is correlated with changes of the optical indices at the interfaces according to Snell law. In order to ascertain whether vibrational changes are correlated to interactions at the solid/liquid interface, optical effects have to be firstly determined.

The calculation of s- and p-polarized spectra of a thin montmorillonite layer deposited on a Ge IRE was performed with an estimated thickness of $7 \mathrm{~nm}$ (Fig. 8a). Given that this thickness is below the penetration depth of the evanescent wave, a third semi-infinite layer of either air or water was considered on the top of the sample film. The optical constants of water were determined using the same methods as in the theory section on a thick layer of water. While the position of vibrational bands is strictly identical for both models, clear modification of band intensity is observed for some specific bands. The out-of-plane Si-O stretching located at $1085 \mathrm{~cm}^{-1}$ appears with a higher intensity when water is considered as the third layer. This variation is associated to drastic changes of the mean squared electric field components along the $\mathrm{z}$ axis. Therefore, only dipole moments having non-zero 
component in the $\mathrm{z}$ direction will be affected by changes of the refractive index of the third layer. Unlike the spolarized spectra where only in-plane transition moments are excited, the influence of a water layer instead of air layer can only be observed on the p-polarized spectrum (Fig. 8b). The intensity of the strongly polarized out-ofplane Si-O stretching is enhanced when the film is immersed in water. However, these results have to be considered in a qualitative way. Indeed, the simulation of the thin layer assumed that the orientation of particles is similar to that of the bulk material from which the anisotropic optical constants, $\hat{n}_{x y}$ and $\hat{n}_{z}$ were determined. The discrepancies between the simulated and experimental spectra account for this variation of particle organisation which is well reflected in the intensity of the in plane and out-of-plane Si-O band.

\section{CONCLUSIONS}

ATR-FTIR technique is a powerful method for studying interactions at the interface between clay surface and solute at the molecular scale. Compared to transmission measurement, ATR spectra appear more complicated because of the significant contribution of optical effects. In this paper, a general formalism to simulate ATR spectra for clay minerals relying on the experimental determination of the optical constants is introduced. In combination to polarized incident radiation, the anisotropic optical constant in the infrared region has been determined for the first time on montmorillonite film. These optical constants allow for the quantitative determination of the sample thickness, and to assess the effect of experimental conditions on the recorded spectra. The formalism being solely based on the classical theory of electromagnetic wave propagation in layered media, differences between the calculated and the experimental spectra allow optical and chemical effects to be discriminated.

\section{ACKNOWLEDGEMENTS}

The CNRS interdisciplinary "défi Needs" funding program (Project DARIUS), is acknowledged for providing financial support for this study. The authors acknowledge financial support from the European Union (ERDF) and "Région Nouvelle Aquitaine". The authors thank J. W. Stucki (Editor in chief), G. Chryssikos (Associate Editor), the anonymous reviewers and C. T. Johnston for their comments that greatly improved the quality of the manuscript.

\section{Conflict of Interest.}

The authors declare that they have no conflict of interest 
Amarasinghe, P. M., Katti, K. S., \& Katti, D. R. (2008). Molecular Hydraulic Properties of Montmorillonite: A Polarized Fourier Transform Infrared Spectroscopic Study. Applied Spectroscopy, 62, 1303-1313.

Aufort, J., Ségalen, L., Gervais, C., Brouder, C., \& Balan, E. (2016). Modeling the attenuated total reflectance infrared (ATR-FTIR) spectrum of apatite. Physics and Chemistry of Minerals, 43, 615-626.

Balan, E., Delattre, S., Roche, D., Segalen, L., Morin, G., Guillaumet, M., et al. (2011). Line-broadening effects in the powder infrared spectrum of apatite. Physics and Chemistry of Minerals, 38, 111-122.

Balan, E., Mauri, F., Lemaire, C., Brouder, C., Guyot, F., Saitta, A. M., et al. (2002). Multiple Ionic-Plasmon Resonances in Naturally Occurring Multiwall Nanotubes: Infrared Spectra of Chrysotile Asbestos. Physical Review Letters, 89, 177401.

Bardwell, J. A., \& Dignam, M. J. (1985). Extensions of the Kramers-Kronig transformation that cover a wide range of practical spectroscopic applications. The Journal of Chemical Physics, 83, 5468-5478.

Berreman, D. W. (1963). Infrared Absorption at Longitudinal Optic Frequency in Cubic Crystal Films. Physical Review, 130, 2193-2198.

Bertie, J. E., \& Lan, Z. (1996). An accurate modified Kramers-Kronig transformation from reflectance to phase shift on attenuated total reflection. The Journal of Chemical Physics, 105, 8502-8514.

Boulet-Audet, M., Buffeteau, T., Boudreault, S., Daugey, N., \& Pézolet, M. (2010). Quantitative Determination of Band Distortions in Diamond Attenuated Total Reflectance Infrared Spectra. The Journal of Physical Chemistry B, 114, 8255-8261.

Brigatti, M. F., Galán, E., \& Theng, B. K. G. (2013). Chapter 2 - Structure and Mineralogy of Clay Minerals. In F. Bergaya, \& G. Lagaly (Eds.), Developments in Clay Science (Vol. 5, pp. 21-81): Elsevier.

Buffeteau, T., Blaudez, D., Péré, E., \& Desbat, B. (1999). Optical Constant Determination in the Infrared of Uniaxially Oriented Monolayers from Transmittance and Reflectance Measurements. The Journal of Physical Chemistry B, 103, 5020-5027.

Dignam, M. J. (1988). Fourier Transform Polarization Spectroscopy. Applied Spectroscopy Reviews, 24, 99-135.

Dignam, M. J., \& Mamiche-Afara, S. (1988). Determination of the spectra of the optical constants of bulk phases via Fourier transform ATR. Spectrochimica Acta Part A: Molecular Spectroscopy, 44, 1435-1442.

Esposito, F., Colangeli, L., \& Palomba, E. (2000). Infrared reflectance spectroscopy of Martian analogues. Journal of Geophysical Research: Planets, 105, 17643-17654.

Farmer, V. C., \& Russell, J. D. (1964). The infra-red spectra of layer silicates. Spectrochimica Acta, 20, 11491173 .

Fraser, R., \& MacRae, T. (1973). Conformation in fibrous proteins and related synthetic polypeptides. New York: Academic Press.

Glotch, T. D., \& Rossman, G. R. (2009). Mid-infrared reflectance spectra and optical constants of six iron oxide/oxyhydroxide phases. Icarus, 204, 663-671.

Grégoire, B., Dazas, B., Hubert, F., Tertre, E., Ferrage, E., Grasset, L., et al. (2020). Orientation measurements of clay minerals by polarized attenuated total reflection infrared spectroscopy. Journal of Colloid and Interface Science, 567, 274-284.

Hansen, W. N. (1965). Expanded formulas for attenuated total reflection and the derivation of absorption rules for single and multiple ATR spectrometer cells. Spectrochimica Acta, 21, 815-833.

Hansen, W. N. (1968). Electric Fields Produced by the Propagation of Plane Coherent Electromagnetic Radiation in a Stratified Medium. Journal of the Optical Society of America, 58, 380-390.

Harrick, N. J. (1965). Electric Field Strengths at Totally Reflecting Interfaces. Journal of the Optical Society of America, 55, 851-857.

Harrick, N. J., \& Beckmann, K. H. (1974). Internal Reflection Spectroscopy. In P. F. Kane, \& G. B. Larrabee (Eds.), Characterization of Solid Surfaces (pp. 215-245). Boston, MA: Springer US.

Hasegawa, T. (2017). Quantitative Infrared Spectroscopy for Understanding of a Condensed Matter: Springer Japan.

Hind, A. R., Bhargava, S. K., \& McKinnon, A. (2001). At the solid/liquid interface: FTIR/ATR — the tool of choice. Advances in Colloid and Interface Science, 93, 91-114.

Huang, J. B., \& Urban, M. W. (1992). Evaluation and Analysis of Attenuated Total Reflectance FT-IR Spectra Using Kramers-Kronig Transforms. Applied Spectroscopy, 46, 1666-1672.

Iglesias, J. E., Ocaña, M., \& Serna, C. J. (1990). Aggregation and Matrix Effects on the Infrared Spectrum of Microcrystalline Powders. Applied Spectroscopy, 44, 418-426.

Johnston, C. T., \& Premachandra, G. S. (2001). Polarized ATR-FTIR Study of Smectite in Aqueous Suspension. Langmuir, 17, 3712-3718.

Karakassides, M. A., Petridis, D., \& Gournis, D. (1997). Infrared reflectance study of thermally treated Li- and Cs-montmorillonites. Clays and Clay Minerals, 45, 649-658.

Lambert, J.-F. (2008). Adsorption and Polymerization of Amino Acids on Mineral Surfaces: A Review. Origins of Life and Evolution of Biospheres, 38, 211-242. 
Larentzos, J. P., Greathouse, J. A., \& Cygan, R. (2007). An ab Initio and Classical Molecular Dynamics Investigation of the Structural and Vibrational Properties of Talc and Pyrophyllite. The Journal of Physical Chemistry C, 111, 12752-12759.

Lefèvre, G., Preočanin, T., \& Lützenkirchen, J. (2012). Attenuated total reflection - Infrared spectroscopy applied to the study of mineral - aqueous electrolyte solution interfaces: a general overview and a case study. In T. Theophile (Ed.), Infrared Spectroscopy: Intech.

Margulies, L., Rozen, H., \& Banin, A. (1988). Use of X-Ray powder diffraction and linear dichroism methods to study the orientation of Montmorillonite clay particles. Clays and Clay Minerals, 36, 476-479.

Milosevic, M. (2004). Internal Reflection and ATR Spectroscopy. Hoboken, New Jersey: John Wiley \& Sons.

Ohta, K., \& Ishida, H. (1988). Comparison Among Several Numerical Integration Methods for Kramers-Kronig Transformation. Applied Spectroscopy, 42, 952-957.

Plaskett, J. S., \& Schatz, P. N. (1963). On the Robinson and Price (Kramers-Kronig) Method of Interpreting Reflection Data Taken through a Transparent Window. The Journal of Chemical Physics, 38, 612-617.

Polubesova, T., \& Chefetz, B. (2014). DOM-Affected Transformation of Contaminants on Mineral Surfaces: A Review. Critical Reviews in Environmental Science and Technology, 44, 223-254.

Ras, R. H. A., Johnston, C. T., Franses, E. I., Ramaekers, R., Maes, G., Foubert, P., et al. (2003). Polarized Infrared Study of Hybrid Langmuir-Blodgett Monolayers Containing Clay Mineral Nanoparticles. Langmuir, 19, 4295-4302.

Ras, R. H. A., Németh, J., Johnston, C. T., Dékány, I., \& Schoonheydt, R. A. (2004a). Infrared reflection absorption spectroscopy study of smectite clay monolayers. Thin Solid Films, 466, 291-294.

Ras, R. H. A., Németh, J., Johnston, C. T., Dékány, I., \& Schoonheydt, R. A. (2004b). Orientation and conformation of octadecyl rhodamine B in hybrid Langmuir-Blodgett monolayers containing clay minerals. Physical Chemistry Chemical Physics, 6, 5347-5352.

Ras, R. H. A., Németh, J., Johnston, C. T., DiMasi, E., Dékány, I., \& Schoonheydt, R. A. (2004c). Hybrid Langmuir-Blodgett monolayers containing clay minerals: effect of clay concentration and surface charge density on the film formation. Physical Chemistry Chemical Physics, 6, 4174-4184.

Ras, R. H. A., Schoonheydt, R. A., \& Johnston, C. T. (2007a). Relation between s-Polarized and p-Polarized Internal Reflection Spectra: Application for the Spectral Resolution of Perpendicular Vibrational Modes. The Journal of Physical Chemistry A, 111, 8787-8791.

Ras, R. H. A., Umemura, Y., Johnston, C. T., Yamagishi, A., \& Schoonheydt, R. A. (2007b). Ultrathin hybrid films of clay minerals. Physical Chemistry Chemical Physics, 9, 918-932.

Roush, T., Pollack, J., \& Orenberg, J. (1991). Derivation of midinfrared (5-25 $\mu \mathrm{m}$ ) optical constants of some silicates and palagonite. Icarus, 94, 191-208.

Schampera, B., \& Dultz, S. (2009). Determination of diffusive transport in HDPy-montmorillonite by H2O-D2O exchange using in situ ATR-FTIR spectroscopy. Clay Minerals, 44, 249-266.

Schampera, B., Šolc, R., Tunega, D., \& Dultz, S. (2016). Experimental and molecular dynamics study on anion diffusion in organically modified bentonite. Applied Clay Science, 120, 91-100.

Stumm, W. (1997). Reactivity at the mineral-water interface: dissolution and inhibition. Colloids and Surfaces A: Physicochemical and Engineering Aspects, 120, 143-166.

Tolstoy, V. P., Chernyshova, I., \& Skryshevsky, V. A. (2003). Handbook of Infrared Spectroscopy of Ultrathin Films: Wiley.

Wilson Edgar, B. (1980). Molecular vibrations : the theory of infrared and Raman vibrational spectra. New York: Dover Publ.

Yamamoto, K., \& Ishida, H. (1994). Interpretation of Reflection and Transmission Spectra for Thin Films: Reflection. Applied Spectroscopy, 48, 775-787. 


\section{Figure Captions}

585 Fig. 1 Schematics of the three-phase system. Notations are defined in the text.

586 Fig. 2 a) Normal and b) $40^{\circ}$ off-normal transmission spectra of Na-SWy-3 montmorillonite ed on ZnSe substrate with s- (green) and p- (blue) 587 polarization.

Fig. 3 a) ATR reflectance spectra on a thick Na-SWy-3 montmorillonite film and the determined (b) real part, $n_{2}$ and (c) imaginary part $k_{2}$ of the anisotropic optical constants. The dashed line in Fig. $3 \mathrm{~b}$ represents the maximum $n_{2}$ value permitted to satisfy the total reflection conditions using Ge ATR.

Fig. 4 TO and LO energy loss functions for a) in-plane and b) out-of-plane components. Spectral simulations of a 20 nm thick Na-SWy-3 film under different optical configurations: c) Normal transmission, d) External reflection on dielectric substrate $\left(\mathrm{SiO}_{2}\right)$, e) Off-normal transmission, f) ATR, g) External reflection at grazing angle on metallic substrate (gold). The transmission Fresnel coefficients were taken from (Milosevic 2004)

Fig. 5 Comparison of experimental spectra of variable thickness Na-SWy-3 montmorillonite films on Ge IRE and calculated ones using the three layer model described in the theory section for the same estimated sample thicknesses. From bottom to top, film thickness is $14,22,33$, 63, 120 and $230 \mathrm{~nm}$.

Fig. 6 Calculated s- and p-polarised spectra of Na-SWy-3 Montmorillonite of different thicknesses on Ge IRE. For the sake of clarity, all spectra are normalized to the most intense vibrational band.

600 Fig. 7 Thickness dependence evolution of a) $\mathrm{LO}_{z}$ surface mode frequency from $1085 \mathrm{~cm}^{-1}$ and b) $\mathrm{TO}_{\mathrm{xy}}$ surface mode intensity at $1039 \mathrm{~cm}^{-1}$

601 Fig. 8 a) Calculated and b) Experimental spectra of thin Na-SWy-3 montmorillonite film at the interface between air or water. 
604

605

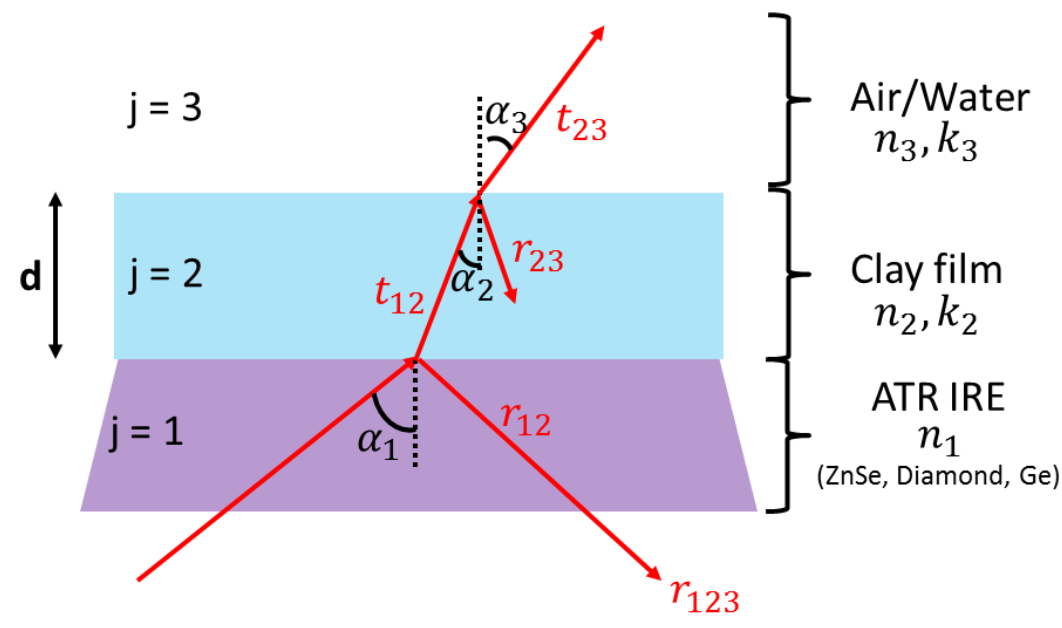

606

Fig. 1 

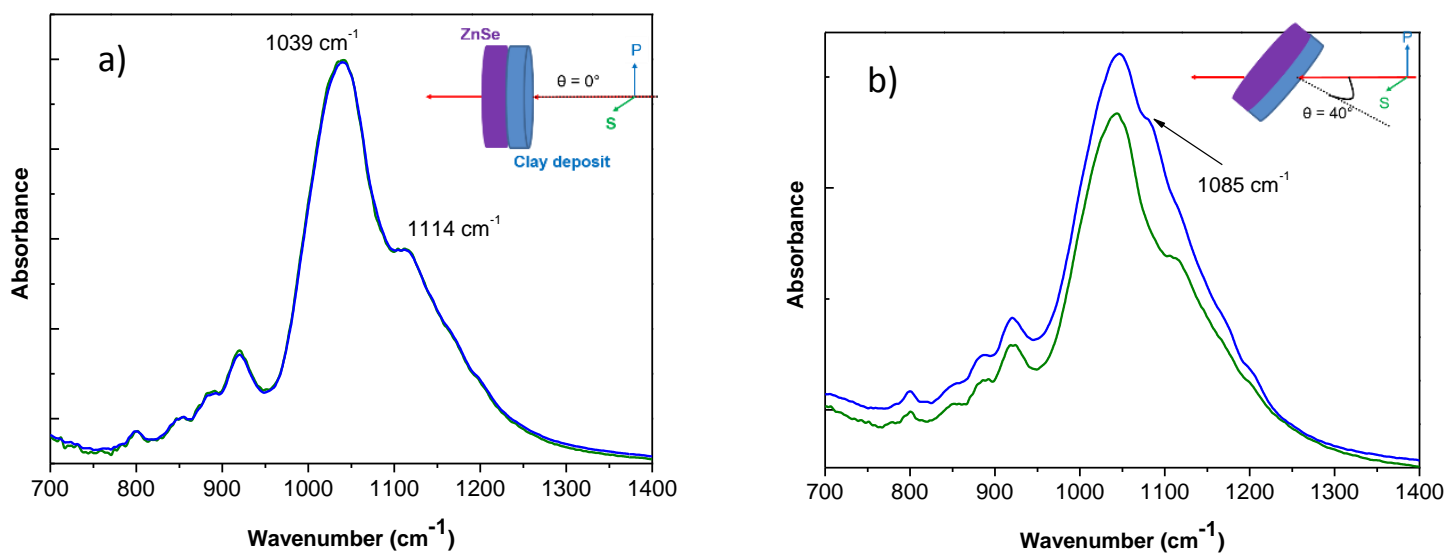

607

608

Fig. 2

609 

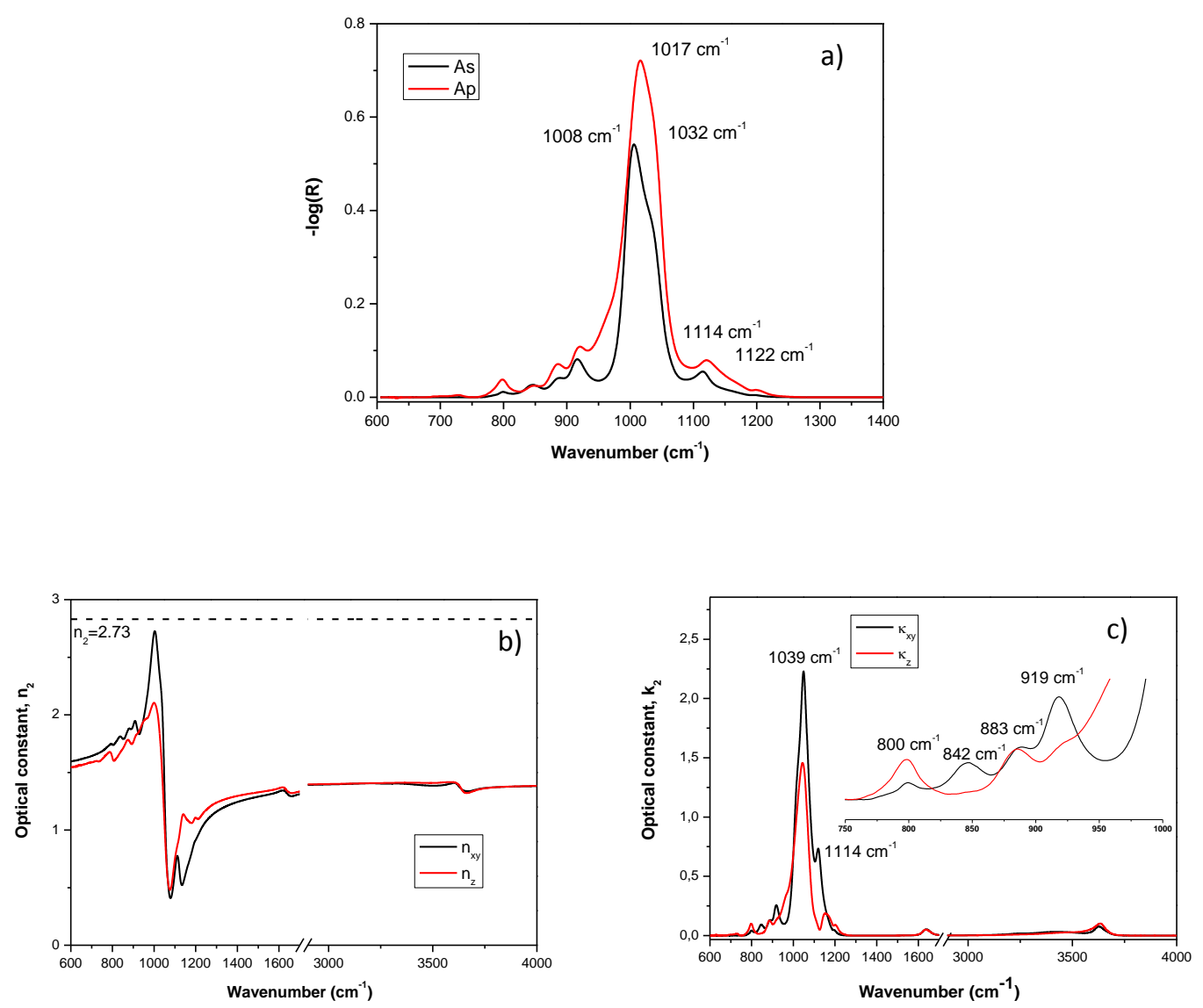

Fig. 3 

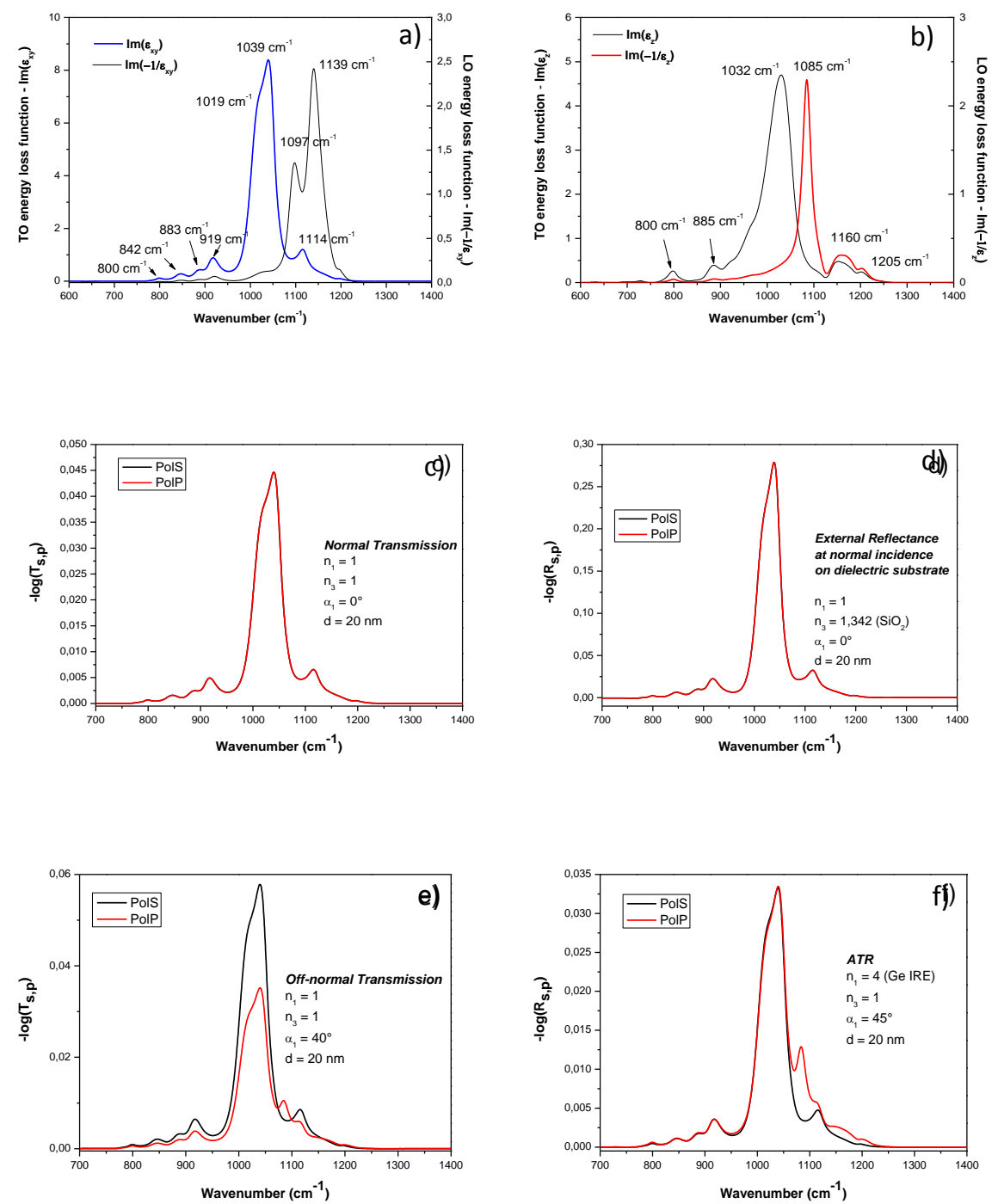

616

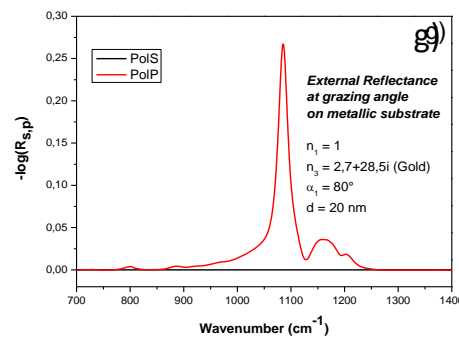

Fig. 4

619 


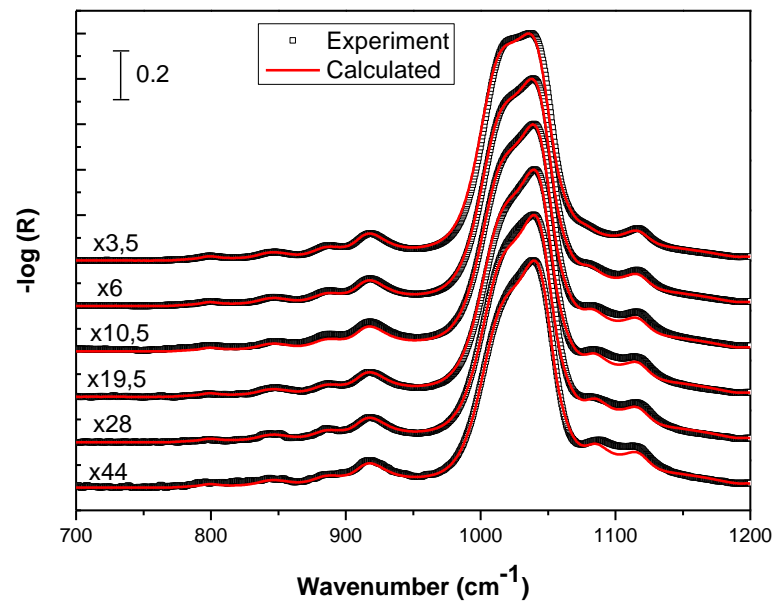

621

622

Fig. 5 


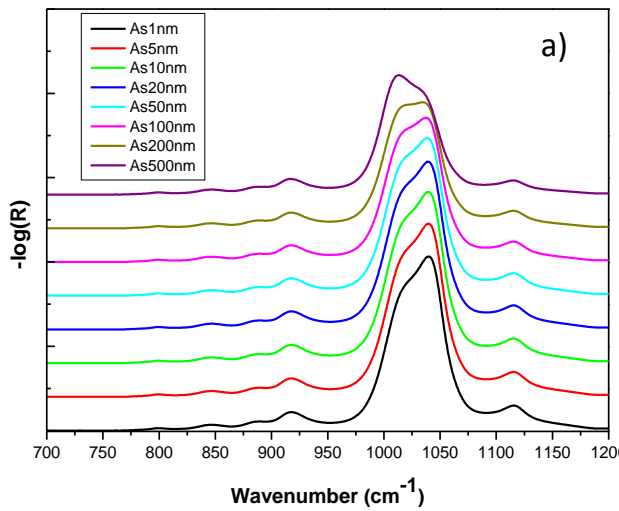

623

624

625

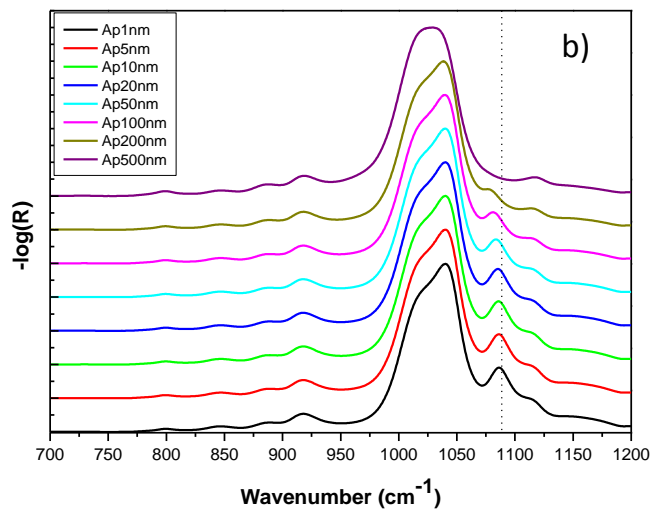

Fig. 6 


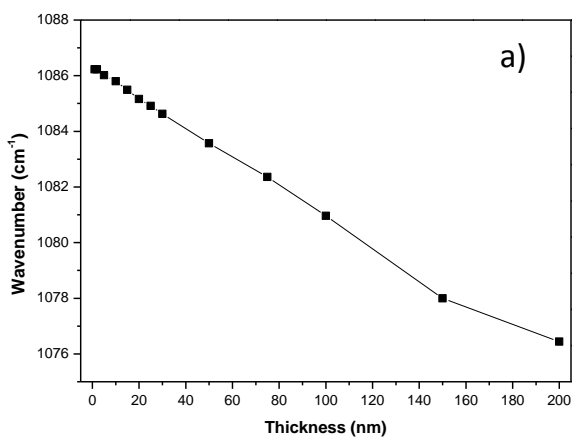

626

627

628

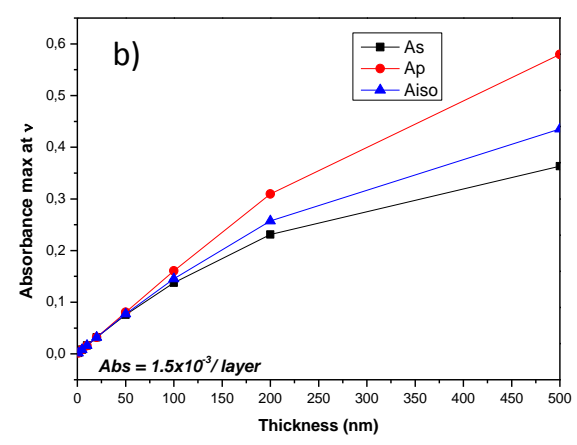

Fig. 7 

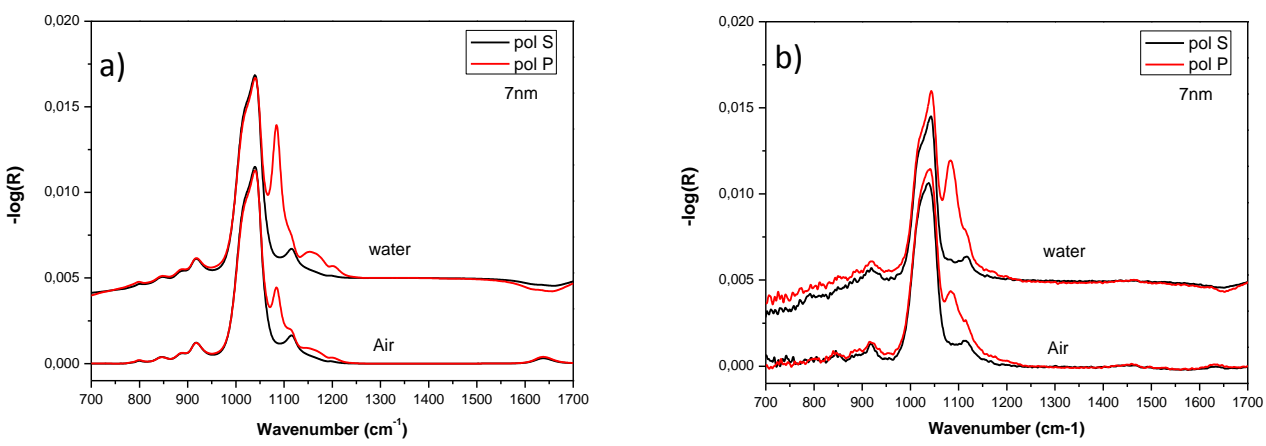

630

631

Fig. 8

632

633

634

635 